\title{
LIQUID-MIRROR TELESCOPE SURVEYS
}

\author{
P.HICKSON \\ University of British Columbia, Dept. Physics \& Astronomy \\ 2219 Main Hall, Vancouver, B.C. V6T1z4 Canada
}

\begin{abstract}
Recent advances in the technology of rotating liquid-mirrors now make feasible the construction of large optical telescopes for dedicated survey programs. Two three-metre-class astronomical telescopes have been built and a six-metre telescope is under construction. These instruments observe in zenith-pointing mode, using drift-scanning CCD cameras to record continuous imaging of a strip of sky typically 20 arcmin wide. This enables them to observe of order 100 square degrees of sky with an integration time of a few minutes per night. Data can be co-added from night to night in order to increase the depth of the survey. Liquid-mirror telescopes are particularly well suited to surveys using broad or intermediate bandwidth filters to obtain photometric redshifts and spectral energy distributions for faint galaxies and quasars.
\end{abstract}

\section{Introduction}

The idea of using a uniformly rotating liquid surface to focus reflected light is an old one [1]. The potential of liquid mirrors for astronomy was realized at least as early as 1850 , with the first example being constructed around 1872 [2]. Well-documented experiments were conducted by Wood [3] who achieved considerable success with a $0.5-\mathrm{m}$ diameter mercury mirror. Using a mechanical bearing, the telescope was able to resolve double stars of 3 arcsec separation. Unfortunately, it was regarded more as a curiosity than a scientific tool. Fifteen years ago, the idea was revisited by Borra [4]. His group at Laval University introduced technological innovations, such as the use of air bearings and thin mercury layers, culminating in a 1.5-m mercury mirror capable of producing diffraction-limited images [5]. The subsequent development, at the University of British Columbia (UBC), of designs and fabrication techniques for large liquid mirrors [6] lead to several fully-functional three-metreclass liquid-mirror telescopes (LMTs) [7-10].

Uniform rotation of a liquid in a homogeneous gravitational field naturally produces a parabolic: surface. The focal length $f$ is simply related to the angular velocity $w$ and gravitational acceleration $g$ by $f=g / 2 w^{2}$. An advantage of a liquid-mirror is that the parabolic surface is accurately maintained by fluid equilibrium despite small deformations of the supporting structure due to mechanical or thermal effects. This greatly simplifies the design and construction of liquid-mirrors, resulting in a cost which is almost two orders of magnitude lower than that of a comparable glass mirror.

A key to the success of mercury mirrors is the thin oxide layer which forms on the surface within a few hours. Virtually transparent, this monomolecular layer is very effective at damping waves and stabilizing the surface. The reflectivity of mercury is between $76 \%$ and $78 \%$ throughout the visible spectrum. Although the reflectivity of fresh aluminum is higher (typically $88 \%$ in the visible and $98 \%$ in the infrared), weathering degrades aluminum coatings, making mercury mirrors competitive in performance at visible and near-infrared wavelengths.

Because the rotation axis must be vertical, these mirrors typically observe in zenith-pointing mode. A prime-focus corrector lens typically provides an instantaneous field of view of order 0.5 deg. Because of the Earth's rotation, images track across this field at the local siderial rate. By aligning the CCD columns with the direction of image motion and continuously reading the CCD at the appropriate rate (drift scanning [11]), this motion is compensated and sharp images result. Except at the equator, there are small departures from linearity in both the direction and speed of 
the images which, if uncorrected, result in degradation of image quality in proportion to the solid angle subtended by the detector. Recent corrector designs incorporate geometric distortion which cancels these effects and produce high-quality images [12].

\section{The UBC-NASA Multiband Survey}

The NASA Orbital Debris Observatory (NODO) employs a 3-m diameter mercury mirror. It is located at an altitude of $2750 \mathrm{~m}$ near Cloudcroft, New Mexico - a dark, dry site having an average of 120 photometric nights per year. The primary purpose of the NODO is to observe space debris in low Earth orbit. However, during the hours when the debris cannot be illuminated by the sun, the telescope may be used for astronomical observations. Since 1996, we have used it to survey a 20-square degree region of the sky at high galactic latitude through a series of intermediate bandwidth filters ([13]). When completed, the survey will provide fluxes in 33 contiguous wavelength bands in the range $455-948 \mathrm{~nm}$ for all detected objects. The data are calibrated by means of spectrophotometric standard stars throughout the survey area established by observations at Kitt Peak National Observatory. The resulting spectral energy distribution (SED's) have resolving power $R \sim 44$ and can provide photometric galaxy redshifts with typical accuracy of $\delta z \simeq 0.01$ [14]. The characteristics of the survey are summarized in Table 1.

The primary scientific goals of this study are to measure the luminosity function, clustering properties and morphological and spectral evolution of galaxies to a redshift $z \sim 0.5$. The survey will be unique in providing flux-calibrated SED's for a large sample of objects. These can be used to investigate, for example, star formation rates for galaxies in a range of environments. We anticipate that redshifts will be obtained for over $10^{4}$ galaxies. These will allow us to reduce contamination by foreground and background galaxies when searching for clusters and groups. Physical properties of these systems can then be investigated and compared with theory. For example, one can measure the frequency of close pairs and thus estimate the variation of the merger rate with redshift $[15,16]$.

A particular advantage of drift-scanned images is the high spatial uniformity which results because pixel sensitivities are averaged over the entire length of the CCD. For the UBC-NASA survey, systematic background variations, after flat-field correction, are less than $0.02 \%$. Because of this the survey is well-suited to the detection of low-surface brightness galaxies. Such galaxies may comprise a high fraction of the population, particularly at low luminosities, so it is particularly important not only to detect them, but also to characterize the selection biases [17]. In a parallel study, broadband observations are being made of the survey region in order to detect and study the structure of objects of low surface brightness.

The survey should also provide a relatively large sample of QSOs, and other objects having strong emission lines, which are quite conspicuous in the multiband data. Because of the wide wavelength range of the survey, it should be relatively free from redshift biases. Simulations indicate that typical QSOs can be reliably identified, and distinguished from stars, when the signal-to-noise ratio of the data is $\sim 10$ or more [18]. The high success rate indicated by the simulations means that statistical studies can proceed without the need for a large amount of follow-up spectroscopy.

The NODO telescope performs very well. At times of good seeing, the resolution of the driftscanned images is limited to 1.0 arcsec FWHM by optical distortion (the corrector lens was not designed for drift scanning because a video camera is used to observe space debris). The corrector is presently being upgraded to remove the distortion. The telescope can be used even in relatively high winds without significant image degradation. The telescope is operated and maintained by a minimal staff, at a cost substantially lower than those of conventional observatories.

\section{The Large Zenith Telescope}

The latest addition to the LMT family is the 6-m Large Zenith Telescope (LZT) under construction near Vancouver. Its dual goals are to develop and test technology needed for the next-generation LMTs, and to extend the multiband drift-scan surveys to fainter magnitudes. The LZT is scheduled to begin observations in early 1998. It is designed to achieve subarcsecond image quality over an 0.3degree fled of view. Equipped with a thinned $2 \mathrm{~K} \times 4 \mathrm{~K}$ CCD camera, it should reach a magnitude limit of $m_{A B} \sim 24$ in a simple night. This will allow it to study the faint-blue galaxy population [20], and achieve sample sizes of order $10^{6}$. The fainter magnitude limit will also facilitate studies of 
TABLE 1. UBC-NASA Multiband Survey

\begin{tabular}{lr}
\hline ra range $(2000):$ & $120000-180000$ \\
dec range $(2000):$ & $325200-330800$ \\
central dec (2000): & 330000 \\
dec width (arcmin): & 16.00 \\
ra length (arcdeg): & 75.48 \\
area (sq deg): & 20.13 \\
pixel size (arcsec): & 0.598 \\
wavelength range (nm): & $455-948$ \\
spectral resolving power: & 44 \\
spatial resolution (arcsec): & 1.5 \\
astrometric accuracy (arcsec): & 1.0 \\
magnitude limit (AB): & 20.4 \\
\hline
\end{tabular}

large-scale structure and the topology of the galaxy distribution, providing a clear discrimination between competing models of structure formation [21].

\section{Future Developments}

Technical solutions employed in the LZT will facilitate the construction of larger liquid mirrors. The air bearing developed for the LZT has sufficient capacity for mercury mirrors of 10-m diameter. The modular construction techniques employed can be readily extended to larger mirrors. A $30-\mathrm{m}$ LMT is not implausible. Alternatively, arrays of 10-m-class telescopes could be contemplated.

A limitation of current LMTs is the need to observe near the zenith. Refracting correctors can provide an instantaneous field of view of about one degree, but larger fields are possible with reflecting correctors. The well-known three-mirror designs of Paul [22] and Baker [23] can provide good image quality over a field of several degrees [24]. Another approach would be the use of adaptive correctors. These contain movable mirrors whose shape can be adjusted to correct aberrations over a range of field angle. Such a corrector could in principle track objects as far as 15 degrees from the zenith [25].

The performance of three-metre LMTs has been proven, and they are regularly used for astronomical observations. The LZT will soon begin operation, providing multi-band data for a million galaxies. Larger LMTs are possible and can provide a cost-effective approach to obtaining large apertures. The potential impact of LMTs, particularly for survey programs, is likely to be substantial.

\section{References}

1. Gibson BK, 1991, J Ray Astr Soc Canada, 85, 158

2. Skey H, 1874, Nature, 10, 274

3. Wood, RW, 1909, Ap J, 29, 164

4. Borra EF, 1982, J Roy Astr Soc Canada, 76, 245

5. Borra EF, Content R, Girard L, Szapiel S, Tremblay LM \& Boily E, 1992, Ap J, 393, 829

6. Hickson, Gibson BK \& Hogg D, 1993, Pub Astro Soc Pacific, 105, 501

7. Hickson P, Borra EF, Cabanac R, Content R, Gibson BK \& Walker GAH, 1994, Ap J, 436, L201

8. Sica RJ, Saragoytchic S, Borra EF, Girad L, Argall S, Sarrow CT \& Flatt S, 1995, Appl Opt, 34, 6925

9. Wurker RF \& Wong A, preprint

10. Potter AE \& Mulrooney MK, 1997, Adv Space Res, 19, 213

11. McGraw JT, Angel JRP \& Sargent TA, 1980, Proc Soc Photo-Opt Instr Eng, 264, 20

12. Hickson $P$ \& Richardson $E H, 1997$, in preparation

13. Hickson $P$ \& Mulrooney MK, 1997, Ap J Suppl, in press

14. Hickson P, Gibson BK \& Callaghan KC, 1994, MNRAS, 267, 911

15. Zepf S \& Koo DC, 1989, Ap J, 337, 34 
16. Woods D, Fahlman GG \& Richer HB, 1995, Ap J, 454, 32

17. Impey C \& Bothun G, 1997, Ann Rev Astr Ap, 35, 267

18. Peri F, Iovino A \& Hickson P, 1997, Science with Liquid-Mirror Telescopes, in press

19. Hickson P, Walker G, Chapman S, de Lapparent V, Borra EF \& Cabanac R, 1997, in preparation

20. Elis RS, 1997, Ann Rev Astr Ap, 35, 389

21. Brandenberger $R, 1997$, private comm.

22. Paul M, 1935, Rev d'Opt, 14, 169

23. Dimitroff GZ \& Baker JG, 1945, Telescopes and Accessories, Blakiston, NY, p. 107

24. Wilson RN, Reflecting Telescope Optics I, Springer-Verlag, Berlin, p. 222

25. Borra EF, Moretto G \& Wang, 1995, Astr. Ap. Suppl., 109, 563, in press 